\title{
Ranolazine Facilitates Termination of Ventricular Tachyarrhythmia Associated With Acute Myocardial Ischemia Through Suppression of Late $I_{\mathrm{Na}}$-Mediated Focal Activity
}

\author{
Takashi Ogawa, MD; Haruo Honjo, MD, PhD; Masatoshi Yamazaki, MD, PhD; \\ Yasunori Kushiyama, MD, PhD; Ichiro Sakuma, $\mathrm{PhD}$; \\ Itsuo Kodama, MD, PhD; Kaichiro Kamiya, MD, PhD
}

\begin{abstract}
Background: Ventricular tachycardia/fibrillation (VT/VF) associated with acute myocardial ischemia is the most common cause of sudden cardiac death, but its underlying mechanisms are incompletely understood. It is hypothesized that late $\mathrm{Na}^{+}$current $(/ \mathrm{Na})$ contributes to arrhythmogenic activity in ischemic myocardium.

Methods and Results: Langendorff-perfused rabbit hearts with regional ischemia in ventricles were optically mapped. Perfusion with ranolazine $(10 \mu \mathrm{mol} / \mathrm{L})$, a selective inhibitor of late $/ \mathrm{Na}$, significantly reduced excitation frequency and facilitated termination of VT/VF induced after occlusion of the left main coronary trunk. The activation pattern during ischemic VT/VF was characterized by breakthrough-type excitations (BEs) from multiple origins, predominantly in the ischemic border zone (BZ) and occasional short-lived rotors. Ranolazine perfusion significantly reduced the incidence of BEs in the BZ. Rotors tended to decrease with progression of ischemia and disappeared after ranolazine perfusion. During constant pacing, ranolazine attenuated ischemia-induced shortening of action potentials in the BZ without affecting conduction velocity, probably due to /kr inhibition. In intact hearts without coronary occlusion, ranolazine $(10 \mu \mathrm{mol} / \mathrm{L})$ terminated aconitine-induced VT by inhibiting focal arrhythmogenic activity in the injection site.
\end{abstract}

Conclusions: Late / Na-mediated focal arrhythmogenic activity plays important roles in the maintenance of ischemic VT/VF in isolated rabbit hearts. Suppression of late $/ \mathrm{Na}$ by ranolazine may be a promising therapeutic strategy to reduce arrhythmic death during the acute phase of myocardial infarction.

Key Words: Action potential; Ischemic ventricular arrhythmia; Late /Na; Optical mapping; Ranolazine

$\mathbf{V}$ entricular tachyarrhythmia, including sustained ventricular tachycardia/fibrillation (VT/VF), associated with acute myocardial ischemia, is the most common immediate cause of sudden cardiac death. ${ }^{1}$ In Japan, arrhythmic mortality in patients with acute myocardial infarction is approximately $14 \%$, and the prevalence of coronary artery disease is increasing in step with the rapid aging of the population., ${ }^{2,3}$ Although percutaneous coronary interventions have been well established as an effective first-line therapy for acute myocardial infarction, therapeutic strategy for ischemia-associated lethal ventricular arrhythmia remains to be developed.

Acute myocardial ischemia is known to cause various changes in the electrophysiological characteristics of ventricular myocardium, and precise mechanisms of acute ischemia-associated arrhythmia are incompletely understood. ${ }^{46}$ A number of recent reports suggest that late $\mathrm{Na}^{+}$ current $\left(I_{\mathrm{Na}}\right)$, which is generated by dysfunctional regulation of $\mathrm{Na}^{+}$channel inactivation, may contribute to arrhythmogenesis in a variety of pathological conditions. ${ }^{7-10}$ Late $I_{\mathrm{Na}}$ is small in normal cardiac myocytes and is enhanced by heart failure, exposure to oxidative stress, hypoxia and ischemia/reperfusion. ${ }^{7-10}$ Late $I_{\mathrm{Na}}$ during the action potential (AP) plateau reduces repolarization reserve and may prolong AP duration (APD), which renders the membrane potential vulnerable for early afterdepolarization (EAD). In addition, increased late $I_{\mathrm{Na}}$-dependent sarcolemmal $\mathrm{Na}^{+}$ entry leads to intracellular $\mathrm{Na}^{+}$accumulation, which in turn produces $\mathrm{Ca}^{2+}$ overload by reduced cellular $\mathrm{Ca}^{2+}$ extrusion and increased $\mathrm{Ca}^{2+}$ entry via the $\mathrm{Na}^{+} / \mathrm{Ca}^{2+}$ exchanger. $\mathrm{Ca}^{2+}$ overload is associated with increased diastolic $\mathrm{Ca}^{2+}$ leak from the sarcoplasmic reticulum resulting in delayed afterdepolarization (DAD). ${ }^{11}$ Moreover, $\mathrm{Ca}^{2+}$ overload activates $\mathrm{Ca}^{2+} /$ calmodulin-dependent protein kinase II (CaMKII), which enhances late $I_{\mathrm{Na}}{ }^{9,11}$ Thus, such a late $I_{\mathrm{Na}}$-mediated positive feedback loop might make a profound contribution

Received February 6, 2017; revised manuscript received April 6, 2017; accepted April 25, 2017; released online May 26, 2017 Time for primary review: 22 days

Department of Cardiovascular Research, Research Institute of Environmental Medicine, Nagoya University, Nagoya (T.O., H.H., M.Y., Y.K., I.K., K.K.); Graduate School of Engineering, The University of Tokyo, Tokyo (I.S.), Japan

Mailing address: Haruo Honjo, MD, PhD, Department of Cardiovascular Research, Research Institute of Environmental Medicine, Nagoya University, Furo-cho, Chikusa-ku, Nagoya 464-8601, Japan. E-mail: honjo@riem.nagoya-u.ac.jp

ISSN-1346-9843 All rights are reserved to the Japanese Circulation Society. For permissions, please e-mail: cj@j-circ.or.jp 


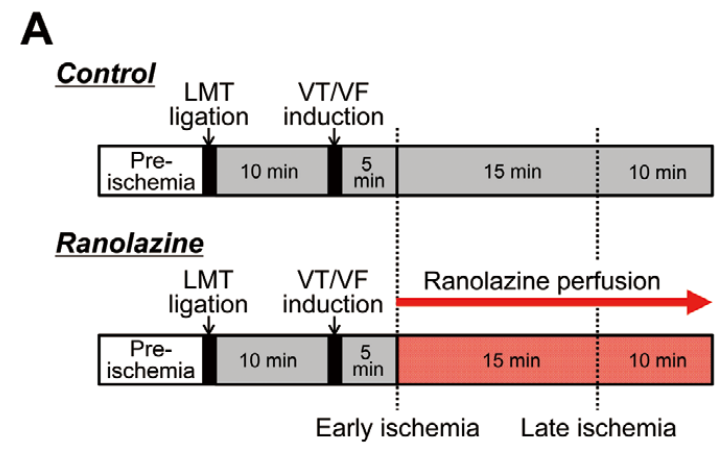

B
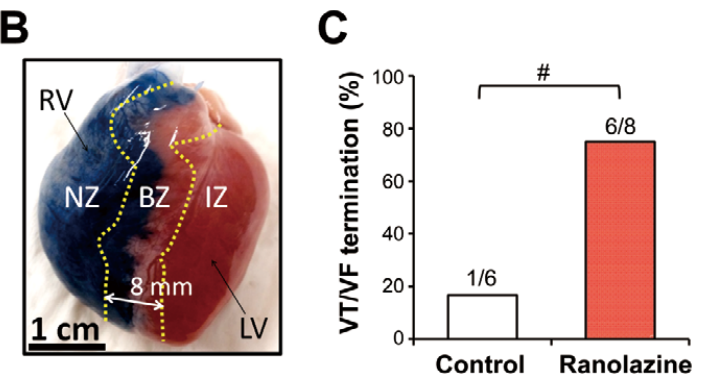

Figure 1. (A) Experimental protocol (see text for explanation). (B) Photograph of a left main coronary trunk (LMT)-ligated heart after injection of Evans Blue into the aorta. IZ, ischemic zone; NZ, non-ischemic zone; BZ, ischemic border zone with a width of $8 \mathrm{~mm}$ between the IZ and IZ; LV, left ventricle; RV, right ventricle. (C) Incidence of ventricular tachycardia/ventricular fibrillation (VT/VF) termination during an observation period of $30 \mathrm{~min}$ after arrhythmia initiation in 6 control and 8 ranolazine-treated hearts. ${ }^{\#}=0.031$ (Fisher's exact probability test).

to arrhythmogenesis.

It is known that late $I_{\mathrm{Na}}$ is inhibited by many class-I antiarrhythmic drugs, such as lidocaine, mexiletine and flecainide, ${ }^{7-9}$ but their antiarrhythmic actions are thought to be conferred primarily through inhibition of peak $I_{\mathrm{Na}}$ during the AP upstroke. In addition, acute amiodarone inhibits both peak and late $I_{\mathrm{Na}}{ }^{8}$ In contrast, ranolazine preferentially blocks late $I_{\mathrm{Na}}\left(\mathrm{IC}_{50}=3-7 \mu \mathrm{mol} / \mathrm{L}\right)$ at the therapeutic range $(1-10 \mu \mathrm{mol} / \mathrm{L})$, although at a higher level, ranolazine suppresses peak $I_{\mathrm{Na}}$ as well (IC50 $>200 \mu \mathrm{mol} / \mathrm{L}) .^{12-15}$ Ranolazine has a relatively minor inhibitory effect on the rapidly activating component of delayed rectifier $\mathrm{K}^{+}$current $\left(I_{\mathrm{Kr}}\right)$ and $H E R G \mathrm{~K}^{+}$current at clinically relevant concentrations. ${ }^{12,13}$ Inhibition of late $I_{\mathrm{Na}}$ by ranolazine has been reported to attenuate AP prolongation, intracellular $\mathrm{Ca}^{2+}$ overload, dynamic instability and regional heterogeneity of electrical activity, as well as arrhythmia in a wide variety of models in which late $I_{\mathrm{Na}}$ was increased by heart failure, exposure to reactive oxygen species or toxins, mutations of $S C N 5 A$ and activation of CaMKII. ${ }^{8,15-17}$

We hypothesized that if late $I_{\mathrm{Na}}$-mediated arrhythmogenic activities contribute to the maintenance of acute ischemia-induced ventricular tachyarrhythmia, then ranolazine should terminate ischemic VT/VF. In the present study, we investigated the effects of ranolazine on VT/VF induced in isolated rabbit hearts with regional ventricular ischemia by quantitative analysis of optical mapping data.

\section{Methods}

The study conforms to the Guide for the Care and Use of Laboratory Animals, and the study protocol was approved by the Institutional Animal Care and Use Committee at Nagoya University.

\section{Optical Mapping Experiments}

The procedures of high-resolution optical mapping are essentially the same as described previously. ${ }^{18,19}$ In brief, male Japanese white rabbits $(1.7-2.0 \mathrm{~kg}, \mathrm{n}=35)$ were anesthetized with thiamylal sodium $(10-15 \mathrm{mg} / \mathrm{kg})$, and heparinized $(250 \mathrm{U} / \mathrm{kg})$. Hearts were quickly removed and were perfused on a Langendorff apparatus with modified KrebsRinger (KR) solution $(\mathrm{NaCl}, 120 \mathrm{mmol} / \mathrm{L} ; \mathrm{KCl}, 4.0 \mathrm{mmol} / \mathrm{L}$; $\mathrm{CaCl}_{2}, 1.8 \mathrm{mmol} / \mathrm{L} ; \mathrm{MgSO}_{4}, 1.3 \mathrm{mmol} / \mathrm{L} ; \mathrm{NaH}_{2} \mathrm{PO}_{4}$, $1.2 \mathrm{mmol} / \mathrm{L}, \mathrm{NaHCO}_{3}, 25.2 \mathrm{mmol} / \mathrm{L}$; glucose, $5.8 \mathrm{mmol} / \mathrm{L}$ ) equilibrated with $95 \% \mathrm{O}_{2}-5 \% \mathrm{CO}_{2}(\mathrm{pH} 7.4)$ at $37^{\circ} \mathrm{C}$. After the hearts were stained with a voltage-sensitive dye, di4-ANEPPS, they were immersed in a tissue bath filled with $\mathrm{KR}$ solution to maintain the temperature at $37^{\circ} \mathrm{C}$. Fluorescence images $(256 \times 256$ pixels $)$ of ventricular epicardial surface were recorded by a high-speed video camera (Fastcam-Max; Photron) at a sampling rate of 1,000 frames/s. To minimize motion artifacts, 2,3-butanedione monoxime (BDM, 20 mmol/L) was added to the perfusate. The ventricular excitation pattern was analyzed by activation isochronal maps drawn with 3-ms intervals. Excitation waves that appeared independently from previous waves were defined as breakthrough-type excitation (BE), and excitation waves that rotated 360 degrees or more were defined as a rotor. Excitation frequency during VT/VF was assessed by fast Fourier transformation of optical action potential signals to obtain dominant frequency (DF). In normalized action potential signals, the time point at $10 \%$ depolarization and $70 \%$ repolarization were identified, and their time interval was defined as action potential duration (APD) at 70\% repolarization (APD 70$)$. An electrocardiogram recording was also made continuously through distant bipolar electrodes to monitor the activity of whole ventricles.

\section{Experimental Protocol}

The experimental protocol is shown in Figure 1A. The left main coronary trunk (LMT) was ligated with nylon thread to create regional ischemia in ventricles $(n=16)$. When sustained VT/VF did not occur spontaneously, electrical stimuli (DC shocks) were applied to induce VT/VF $10 \mathrm{~min}$ after LMT occlusion. In 8 hearts, coronary perfusion with $10 \mu \mathrm{mol} / \mathrm{L}$ ranolazine (Sigma-Aldrich, USA) was started 5 min after initiation of sustained VT/VF, and then the hearts were continuously perfused with ranolazine $(10 \mu \mathrm{mol} / \mathrm{L})$ during an observation period up to $30 \mathrm{~min}$ after arrhythmia initiation (ranolazine group, $n=14$ ). Two hearts that also exhibited sustained VT/VF were perfused with E4031 $(0.01 \mu \mathrm{mol} / \mathrm{L})$, and 6 hearts without drug perfusion served as controls. At the end of each experiment, Evans Blue was injected into the coronary artery to identify the ischemic and non-ischemic zones (IZ, NZ, respectively, Figure 1B). The ischemic border zone (BZ) was defined as a peripheral ischemic area with a width of $8 \mathrm{~mm}$.

\section{Assessment of Ischemia-Dependent Electrophysiological Changes}

Acute ischemia-dependent changes in electrophysiological 
properties and their modification by ranolazine were investigated in hearts with LMT ligation $(n=12)$. In this study, complete atrioventricular block was made by atrioventricular node ablation, and constant stimulation (pacing cycle length, PCL, $400 \mathrm{~ms}$ ) was applied from the NZ. The $\mathrm{APD}_{70}$ (average of 16 sites covering a $5 \times 5-\mathrm{mm}$ square area) and conduction velocity $(\mathrm{CV})$ in each zone were measured before and after LMT ligation. Six hearts were continuously perfused with $10 \mu \mathrm{mol} / \mathrm{L}$ ranolazine after LMT ligation (ranolazine group), while the other 6 hearts were perfused with normal perfusate without ranolazine (control group). The effects of E-4031 on APD 70 were assessed in 3 hearts without coronary occlusion under constant pacing (PCL, $400 \mathrm{~ms}$ ).

\section{Aconitine-Induced VT}

Aconitine is known to enhance late $I_{\mathrm{Na}}$ by modulating $\mathrm{Na}^{+}$ channel inactivation. ${ }^{20}$ The effects of ranolazine on aconitine-induced VT were investigated in 4 hearts without coronary occlusion. Aconitine $(50 \mu \mathrm{L}$ of $0.4 \mathrm{mmol} / \mathrm{L}$ solution; Sigma Aldrich, USA) was injected directly into the left ventricular subepicardial muscle. Three hearts were perfused with $10 \mu \mathrm{mol} / \mathrm{L}$ ranolazine during aconitineinduced sustained VT, while in the remaining 1 heart, aconitine-induced VT was observed without interventions.

\section{Statistical Analysis}

Data are presented as means or means \pm SE. Comparison of arrhythmia termination rate with and without ranolazine perfusion was evaluated using Fisher's exact probability test. For comparison of multiple group data, ANOVA with Turkey-Kramer correction was used. $\mathrm{P}<0.05$ was considered statistically significant.

\section{Results}

Effects of Ranolazine on Ischemic Ventricular Arrhythmia In 6 control hearts, sustained VT/VF was induced by DC shocks after LMT ligation, and VT/VF terminated spontaneously in 1 heart $(17 \%) \sim 13 \mathrm{~min}$ after its initiation, while in the remaining 5 hearts, VT/VF was maintained during the observation period up to $30 \mathrm{~min}$ after arrhythmia initiation (Figure 1C). In the ranolazine group $(n=8)$, sustained VT/VF occurred spontaneously in 1 heart and was induced in 7 hearts after LMT occlusion. Sustained VT/VF terminated in 6 out of 8 hearts $(75 \%, \mathrm{P}=0.031)$ during perfusion with ranolazine (Figure 1C). The average time from the onset of ranolazine perfusion to arrhythmia termination was $13.9 \pm 7.7 \mathrm{~min}(\mathrm{n}=6)$.

\section{Excitation Patterns During Ischemic Ventricular Arrhythmia}

The pattern of excitation wave propagation during arrhythmia was analyzed with the aid of a high-resolution optical mapping technique. Figure 2 shows data for local excitation frequency during ischemic VT/VF. The DF of local excitation was determined by fast Fourier transformation of optical action potential signals, and the pattern of spatial DF distribution during VF was illustrated. In a representative example shown in Figure 2A, higher DF was observed in the BZ (highest DF [DFmax $]$ in $\mathrm{BZ}, 13.0 \mathrm{~Hz}$ ) compared with that in the IZ (DFmax in IZ, $11.9 \mathrm{~Hz})$ and in the NZ (DF $\max$ in NZ, 10.6 Hz). Figure 2B summarizes regional $\mathrm{DF}_{\max }$ from 6 control and 8 ranolazine-perfused hearts at the early ( $\sim 15 \mathrm{~min}$ after LMT occlusion) and later $(\sim 30 \mathrm{~min})$ ischemic phases. In control hearts, DF $\max$ in the

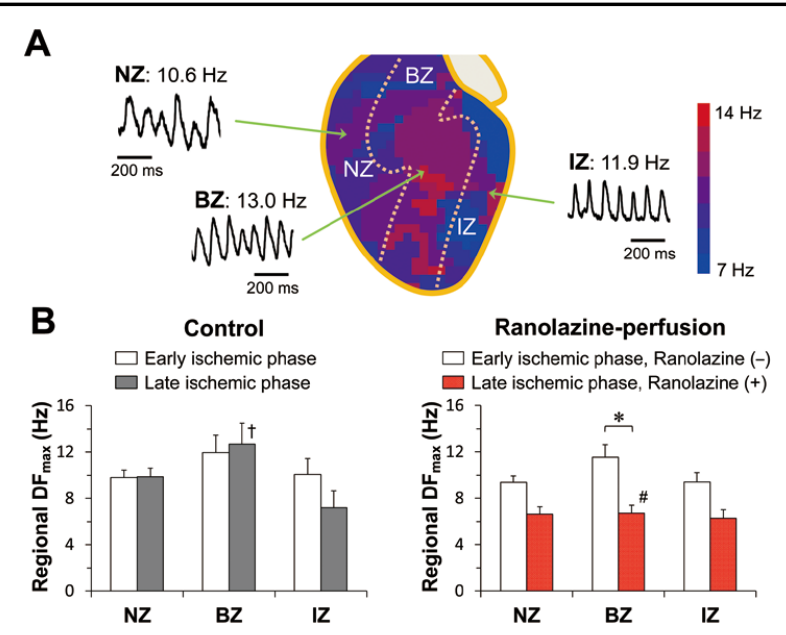

Figure 2. Local excitation frequency during ischemic VTNF (A) A representative dominant frequency (DF) map in a control heart, with optical action potential (AP) signals recorded from three sites in the non-ischemic zone (NZ), border zone (BZ) and ischemic zone (IZ) where their DF values were highest $\left(\right.$ DF $_{\max }$ ) in each zone. (B) Comparison of DFmax in the NZ, BZ and IZ. Data were obtained from 6 control hearts (Left) at the early ischemic phases ( $\sim 15$ after LMT ligation) and at the late ischemic phase ( 30 min after LMT ligation), and from 8 ranolazine-perfused hearts (Right) at the early ischemic phase before ranolazine application and at the late ischemic phase during ranolazine perfusion. ${ }^{\dagger} \mathrm{P}<0.05$ vs. IZ; ${ }^{*} \mathrm{P}<0.05$ vs. early ischemic phase; \#P<0.05 vs. control. Abbreviations as in Figure 1.

BZ tended to be higher than that in the IZ and NZ, indicating that the BZ drives long-lasting sustained arrhythmia during acute myocardial ischemia. In ranolazine-perfused hearts, $\mathrm{DF}_{\max }$ in the BZ was significantly decreased at the late ischemic phase during ranolazine perfusion compared with that at the early ischemic phase before ranolazine application. Thus, there was a significant decrease in $\mathrm{DF}_{\max }$ in the $\mathrm{BZ}$ at the late ischemic phase in ranolazine-perfused hearts compared with controls without ranolazine treatment. These results suggest that ranolazine suppresses arrhythmogenic electrical activity mainly in the BZ, which maintains ischemic VT/VF.

Figure 3 shows ventricular excitation patterns during ischemic VT/VF. Representative isochronal activation maps obtained from a heart at the early ischemic phase (Figure 3A) show a complex and disorganized pattern with BEs emanating from multiple origins, distorted lines of functional conduction block and occasional short-lived rotors. The BE origins and rotors were located predominantly in the BZ. In this heart, ischemic VT/VF terminated during ranolazine perfusion, and activation maps obtained at the late ischemic phase before arrhythmia termination show a more organized pattern with BEs from a single site in the BZ and without rotors (Figure 3B). In contrast, activation maps from another control heart without ranolazine perfusion at the late ischemic phase remained disorganized with multiple BEs, and rotors were rarely detected (Figure 3C). In this control heart, ischemic VT/VF persisted until the end of the experiment.

Figure 4 summarizes the incidence of BEs and rotors during a unit of time (1s) in 6 control (Figure 4, Left) and 8 ranolazine-perfused hearts (Figure 4, Right). In controls, 
A Early ischemic phase, Ranolazine (-)
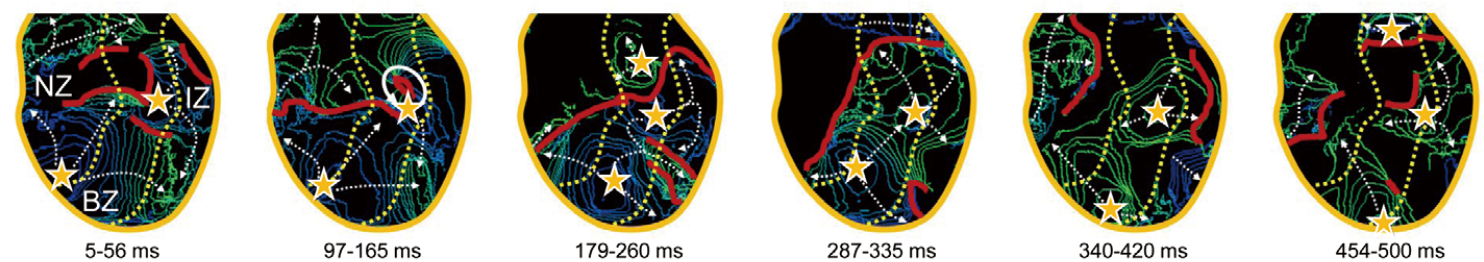

\section{B Late ischemic phase before VT/VF termination, Ranolazine (+)}
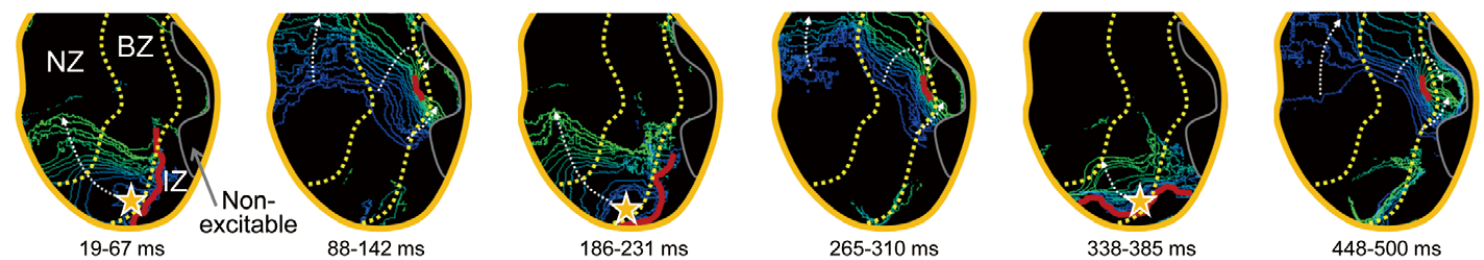

C Late ischemic phase, Ranolazine (-)
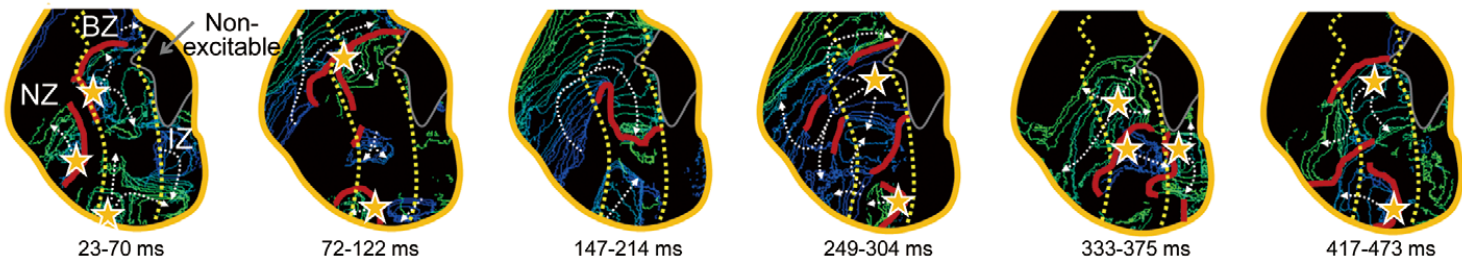

Figure 3. Sequential isochronal activation maps during ischemic VT/VF. Superimposed 3-ms isochrones with varying colors, from blue for early wavefronts to green for later ones, are illustrated in each map. Data were obtained from a heart at the early ischemic phase before ranolazine application $(\mathbf{A})$ and at the late ischemic phase during ranolazine perfusion (B), and from another control heart without ranolazine perfusion at the late ischemic phase $(\mathbf{C})$. Red lines, functional conduction block with local conduction velocity $(\mathrm{CV})<3 \mathrm{~cm} / \mathrm{s}$; yellow stars, the initiating site of breakthrough-type excitations (BEs); white arrows, rotors. Non-excitable tissue in the $\mathrm{IZ}$ is delineated by a gray line. Abbreviations as in Figure 1.
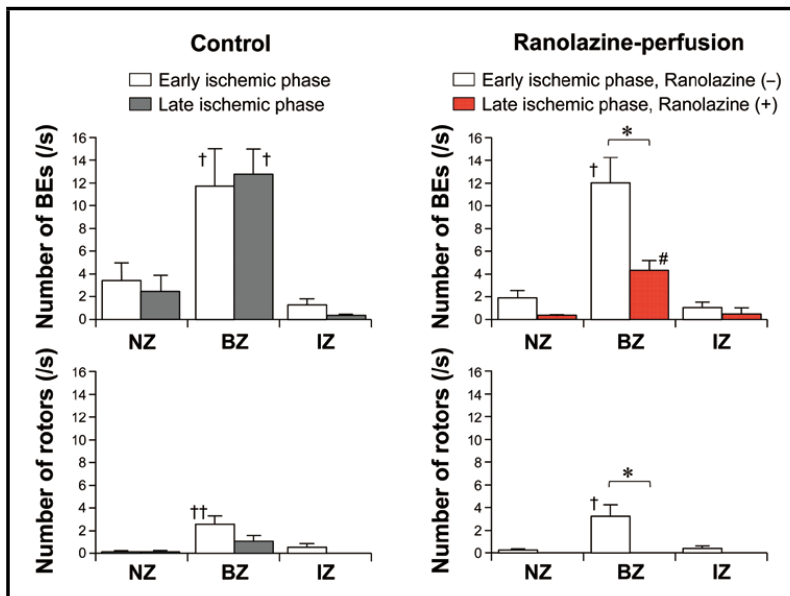

Figure 4. Incidence of BEs and rotors (per 1s) during ischemic VT/NF. Data were obtained from 6 control hearts (Left) at the early and late ischemic phases, and from 8 ranolazineperfused hearts (Right) at the early ischemic phase before ranolazine application and at the late ischemic phase during ranolazine perfusion. ${ }^{\mathrm{P}} \mathrm{P}<0.05 \mathrm{vs}$. IZ and NZ; ${ }^{\dagger+P}<0.05$ vs. NZ; ${ }^{*} \mathrm{P}<0.05$ vs. early ischemic phase; ${ }^{\mathrm{P}}<0.05$ vs. control. Abbreviations as in Figures 1,3. the incidence of BEs in the $\mathrm{BZ}$ was significantly higher than in the other regions at the early ischemic phase, and this pattern was maintained at the late ischemic phase. In ranolazine-perfused hearts, the incidence of BEs was significantly decreased at the late ischemic phase during ranolazine perfusion from that at the early ischemic phase before ranolazine application. Thus, the incidence of BEs in the BZ during ranolazine perfusion was significantly less compared with that in controls without ranolazine perfusion. The average number of rotors detected during ischemic VT/VF was much lower compared with that of BEs. In controls, the incidence of rotors tended to decrease with progression of ischemia. All rotors were short-lived, and the average number of consecutive rotations was $1.3 \pm 0.2$. Rotors were not detected in ranolazine-perfused hearts at the late ischemic phase.

\section{Effects of Ranolazine on Ischemia-Induced Electrophysiological Changes}

Acute ischemia-dependent changes in APD and CV and their modifications by ranolazine were investigated in hearts with LMT ligation under constant pacing (PCL, $400 \mathrm{~ms}$; Figure 5). Figure 5A shows examples of superimposed optical AP signals recorded from a control heart and 


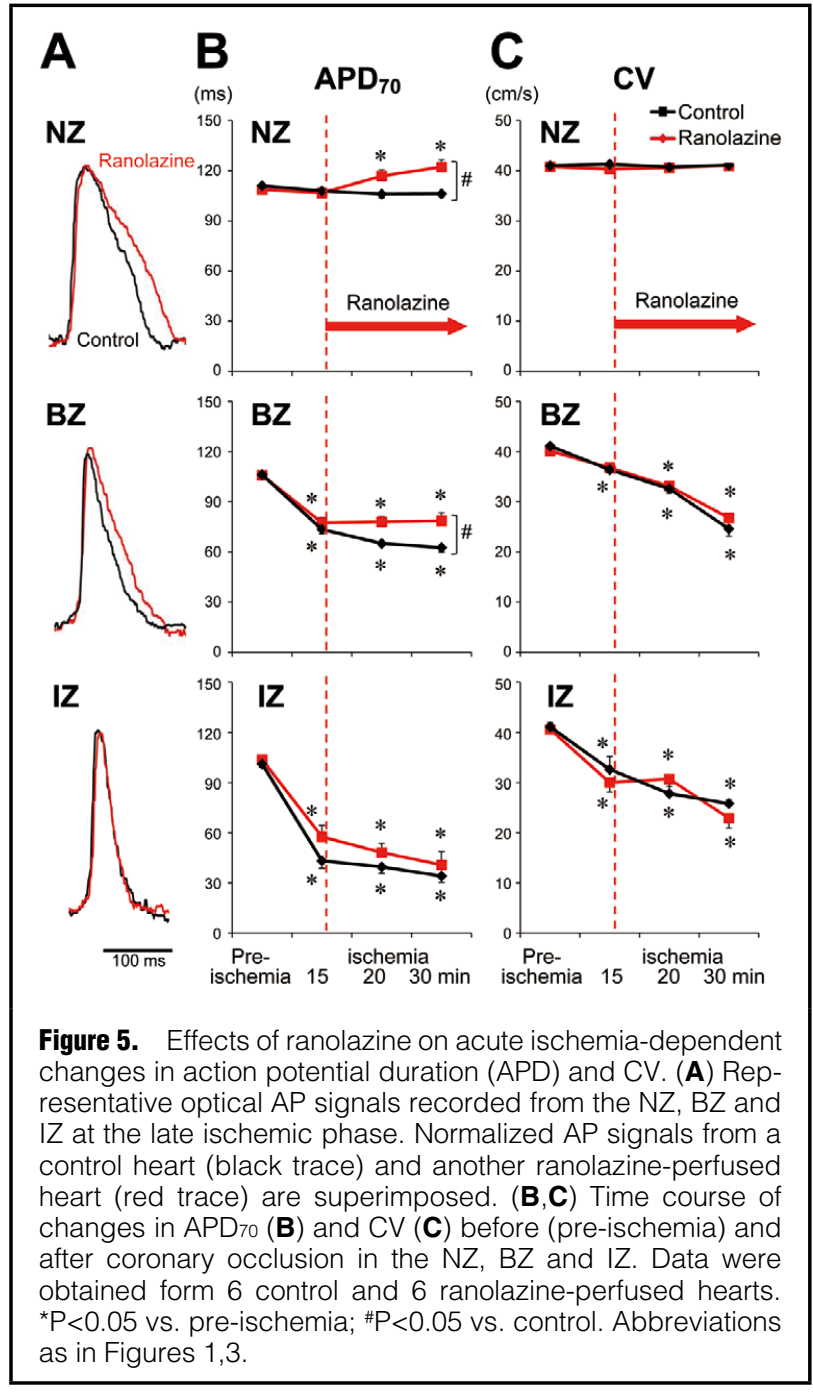

from another ranolazine-perfused heart at the late ischemic phase. In the control heart, there was a gradual APD shortening from the NZ to the IZ, reflecting ischemiadependent metabolic changes. APD in the NZ and the BZ of the ranolazine-perfused heart was longer compared with controls. Figure 5B shows the time-course of changes in $\mathrm{APD}_{70}$ before and after coronary occlusion in 6 control and 6 ranolazine-perfused hearts. In the controls, APD 70 was shortened with the progress of ischemic changes in the $\mathrm{IZ}$ and the BZ. Ranolazine perfusion significantly prolonged $\mathrm{APD}_{70}$ in the NZ and the BZ compared with controls, probably due to $I_{\mathrm{Kr}}$ inhibition by ranolazine. APD 70 in the IZ was unaffected. The resting membrane potential is expected to be affected by acute ischemia, but this cannot be evaluated in optical AP data. CV was progressively decreased in the IZ and the BZ after coronary occlusion, and ranolazine perfusion had no appreciable effects on these ischemia-dependent changes in $\mathrm{CV}$.

\section{Effects of E-4031 on Action Potential and Ischemic Ventricular Arrhythmia}

The selective blockade of $I_{\mathrm{Kr}}$ by E-4031 $(0.01 \mu \mathrm{mol} / \mathrm{L})$ prolonged $\mathrm{APD}_{70}$ by $19 \pm 1 \%(\mathrm{n}=3)$ under constant pacing (PCL, $400 \mathrm{~ms}$ ) in ventricles of intact hearts (Figure S1).

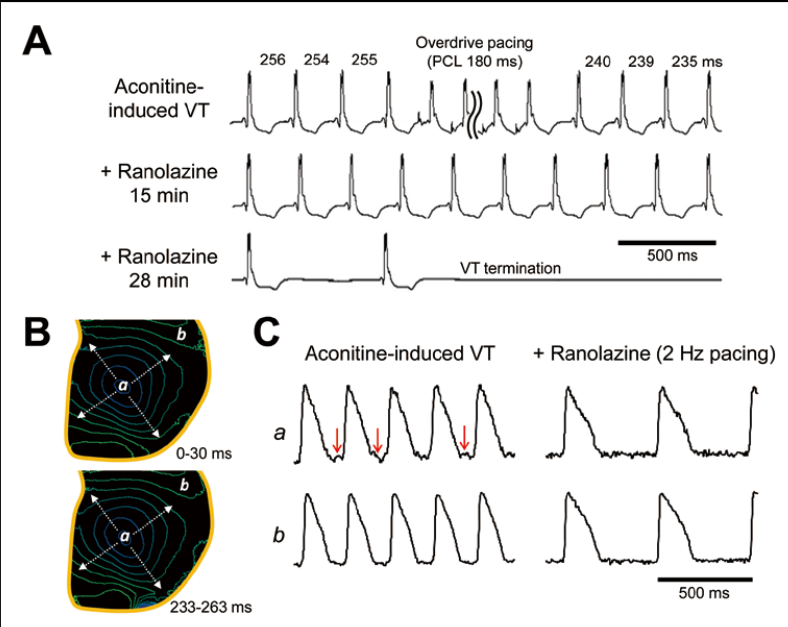

Figure 6. Effects of ranolazine on aconitine-induced VT. (A) Electrocardiograms of aconitine-induced VT before (Top) and during ranolazine perfusion (Middle and Bottom). Numbers above the trace indicate VT cycle length before and after application of overdrive pacing (pacing cycle length, $180 \mathrm{~ms}$; 100 pulses). (B) Isochronal activation maps during aconitineinduced VT. (C) Optical AP signals recorded from 2 sites (a, aconitine injection site and $b$, peripheral site in (B) during aconitine-induced VT (Left) and during $2 \mathrm{~Hz}$ pacing after VT termination in the presence of ranolazine (Right). Red arrows indicate delayed after depolarization (DAD)-like small humps in the resting potential. Abbreviations as in Figures 1,2.

Two hearts that showed sustained VT/VF after LMT occlusion were perfused with E-4031 $(0.01 \mu \mathrm{mol} / \mathrm{L})$. Ischemic VT/VF persisted in these hearts for more than $1 \mathrm{~h}$ after E-4031 application. Activation maps at the late ischemic phase during E-4031 perfusion show a disorganized pattern with multiple BEs and infrequent short-lived rotors (average $\mathrm{DF}_{\max }, 10.6 \mathrm{~Hz}$ in the BZ, Figure $\mathrm{S1}$ ).

\section{Effects of Ranolazine on Aconitine-Induced Ventricular Tachycardia}

In a separate series of experiments, the effects of ranolazine on late $I_{\mathrm{Na}}$-mediated triggered activities were investigated in hearts without coronary occlusion ( $n=4$, Figure 6). Late $I_{\text {Na-mediated triggered activities were induced by an intra- }}$ muscular injection of aconitine. The aconitine injection induced monomorphic VT $(4.8 \pm 0.4 \mathrm{~Hz}, \mathrm{n}=4$, Figure 6A), which persisted for more than $1 \mathrm{~h}$ without interventions. Overdrive pacing (PCL, $180 \mathrm{~ms}, 100$ pulses) applied caused a transient acceleration of VT. Isochronal activation maps during sustained VT show BE-type focal activation, initiated from the aconitine injection site (Figure 6B). In optical AP signals recorded from the injection site, DAD-like small humps were observed during electrical diastole (Figure 6C). Perfusion with ranolazine $(10 \mu \mathrm{mol} / \mathrm{L})$ resulted in a progressive increase in the VT cycle length and subsequent termination of VT in all 3 hearts tested. In addition, ranolazine perfusion abolished diastolic humps in AP signals. The average time from the onset of ranolazine perfusion to VT termination was $23.7 \pm 3.3 \mathrm{~min}(\mathrm{n}=3)$.

\section{Discussion}

The major findings obtained from the present study are 
summarized as follows: (1) ranolazine perfusion facilitated termination of ischemic VT/VF induced after coronary occlusion in isolated rabbit hearts; (2) during ischemic VT/ $\mathrm{VF}$, focal activity with multiple origins and occasional short-lived rotors were observed in the BZ where DF of local excitation was highest. Ranolazine perfusion significantly suppressed focal activity in the BZ. Short-lived rotors became less frequent with progression of ischemia, and disappeared after ranolazine perfusion; (3) under constant pacing, ranolazine reduced ischemia-induced APD shortening in the BZ without affecting CV; and (4) in the intact heart without ischemia, ranolazine effectively terminated aconitine-injection induced monomorphic VT by suppressing focal arrhythmogenic activity in the injection site.

\section{Arrhythmogenic Mechanisms During Acute Myocardial Ischemia}

High-resolution optical mapping analysis in the present study shows that ischemic VT/VF induced in Langendorffperfused rabbit hearts are maintained mainly by focal activity with multiple origins and functional re-entry located predominantly in the BZ. These findings are consistent with the results of earlier experimental studies of various animal species. ${ }^{46}$ It has been reported that ischemic ventricular arrhythmia has temporally 2 distinct phases: phase 1, from immediately after coronary occlusion to $\sim 30 \mathrm{~min}$, and phase 2 , beginning from $\sim 1.5 \mathrm{~h}$ to $\sim 72 \mathrm{~h}$ after occlusion. ${ }^{46}$ Phase 1 arrhythmias are known to occur in association with ischemia-induced changes in metabolism and ion homeostasis, and both ectopic focal mechanisms and functional re-entry are thought to be involved. ${ }^{\mathbf{4}} \mathbf{6}$ APD shortening in ischemic tissues in the present study reflects increased net outward current, resulting from activation of ATP-sensitive $\mathrm{K}^{+}$current (IK,ATP) and reduction of L-type $\mathrm{Ca}^{2+}$ current $\left(I_{\mathrm{Ca}, \mathrm{L}}\right)$ in response to depletion of ATP and/or building up of ADP and inorganic phosphate, accumulation of anaerobic glycolysis products and intracellular acidification. Ischemia-induced conduction slowing can be explained, partly by extracellular $\mathrm{K}^{+}$accumulationdependent depolarization of the resting potential, which causes voltage-dependent inactivation of $\mathrm{Na}^{+}$channels, and partly by intercellular gap junctional uncoupling. These changes in APD and CV abbreviate excitation wavelength predispose to formation of functional reentry. In addition, intracellular $\mathrm{Ca}^{2+}$ overload is well-known to contribute to phase 1 arrhythmia by the mechanism of focal arrhythmogenic activity, such as triggered activity and abnormal automaticity. ${ }^{46,11} \mathrm{Ca}^{2+}$ overload is produced under ischemic metabolism, partly via intracellular acidificationdependent reduction of the $\mathrm{Na}^{+} / \mathrm{H}^{+}$exchanger activity and resulting $\mathrm{Na}^{+}$overload-induced reduction of $\mathrm{Ca}^{2+}$ extrusion by the $\mathrm{Na}^{+} / \mathrm{Ca}^{2+}$ exchanger. ${ }^{46,11}$

\section{Effects of Ranolazine on Ischemia-Induced Arrhythmogenic Activities}

The data from the present study demonstrate that ranolazine perfusion significantly facilitated the termination of ischemic VT/VF in isolated rabbit hearts. This indicates that late $I_{\text {Na }}$ plays important roles in phase 1 arrhythmogenesis, especially for the maintenance of sustained VT/ VF. Ranolazine-induced reduction in late $I_{\mathrm{Na}}$ attenuates intracellular $\mathrm{Na}^{+}$accumulation and suppression of $\mathrm{Ca}^{2+}$ overload-induced DADs. ${ }^{7-11}$ In addition, it is possible that the reduction in late $I_{\mathrm{Na}}$ may prevent enhanced automaticity in depolarized myocardium by suppression of diastolic depolarization. Our optical mapping data demonstrate that ranolazine significantly reduced the incidence of focal activity in the BZ during phase 1 ischemia. In addition, the same concentration of ranolazine abolished aconitineinduced DAD-like membrane potential humps and terminated aconitine-induced monomorphic VT in intact hearts. These results support the hypothesis that the antiarrhythmic action of ranolazine against ischemic ventricular arrhythmia is mediated by the suppression of late $I_{\mathrm{Na}}$-dependent focal arrhythmogenic activity.

Breakthrough-type excitations observed during ischemic VT/VF might reflect intramural re-entry. In ischemic myocardium with reduced excitability, 3-dimentional scroll waves with a curved filament could be generated. ${ }^{21,22}$ However, such a scroll with an elongated filament is highly unstable and quickly degenerates into multiple scrolls with shorter filaments, which appear as rotors on the epicardial surface. Therefore, the result that the number of rotors was much lower than that of BEs during VT/VF is inconsistent with the possibility that BEs reflect intramural re-entry.

Action potential is expected to be shortened by ranolazine through the reduction in persistent inward $I_{\text {Na. }}$ However, our data show that APD in the NZ and BZ was significantly prolonged during ranolazine perfusion compared with controls. The ranolazine-induced APD prolongation is thought to be a result of the ranolazine's inhibitory action on $I \mathrm{Kr},{ }^{12,13}$ and may make an additional contribution to VT/VF termination through prevention of functional re-entry formation. An earlier experimental study in in vivo pigs showed that ranolazine suppressed the ischemia-induced increase in T-wave alternans, ${ }^{23}$ and such improvement of dynamic instability may also be involved in suppression of stable rotor formation. However, it is unlikely that the suppression of functional re-entry is the principal mechanism for ranolazine to facilitate termination of ischemic VT/VF in the present study. At the late phase of ischemia, short-lived rotors were rarely observed even in control hearts without ranolazine perfusion and, therefore, rotors may not be responsible for the maintenance of ischemic VT/VF in the present rabbit model.

In addition, selective blockade of $I_{\mathrm{Kr}}$ by $0.01 \mu \mathrm{mol} / \mathrm{L}$ E-4031, which caused an APD prolongation under constant pacing (PCL, $400 \mathrm{~ms}$ ) to the same degree as $10 \mu \mathrm{mol} / \mathrm{L}$ ranolazine, failed to terminate ischemic $\mathrm{VT} / \mathrm{VF}$ in Langendorff-perfused rabbit hearts with LMT ligation. This result further supports the hypothesis that the late $I_{\mathrm{Na}}$-mediated focal arrhythmogenic activity is responsible for the maintenance of ischemic VT/VF, and the inhibitory action of ralorazine on the late $I_{\mathrm{Na}}$ plays a major role in its antiarrhythmic effects on ischemic VT/VF, at least in isolated rabbit hearts with coronary occlusion, although epicardial optical mapping cannot completely eliminate the possibility that micro-re-entry may contribute to the maintenance of ischemic ventricular arrhythmia.

Ranolazine was developed as an antianginal agent, and the antianginal effects of ranolazine are mainly related to its inhibition of late $I_{\mathrm{Na}}$, which improves diastolic compliance of ventricles through a reduction of intracellular $\mathrm{Ca}^{2+}$, thereby increasing coronary artery blood flow. ${ }^{24}$ It is possible that the anti-ischemic effects of ranolazine may also play a role in suppressing arrhythmogenic activities in the BZ.

\section{Clinical Implications}

A number of recent randomized case-controlled clinical 
studies have demonstrated that ranolazine is effective in reducing angina with favorable safety in patients with chronic angina. ${ }^{25-28}$ The ECG data from one of these studies, the MERLIN-TIMI (Metabolic Efficiency with Ranolazine for Less Ischemia in Non-ST Elevation Acute Coronary Syndrome: Thrombosis in Myocardial Infarction) 36 trial, showed that the incidence of ventricular and atrial tachyarrhythmia was significantly decreased by treatment with ranolazine. ${ }^{28}$ Experimental studies also demonstrated that pre-treatment of rat and pig hearts with ranolazine reduced vulnerability to VF and prevented sustained VT/VF in the setting of acute ischemia and/or ischemia/reperfusion. . 23,29,30 $^{2}$ A selective late $I_{\mathrm{Na}}$ inhibitor, GS-458967, has been reported to reduce ischemia-induced T-wave alternans in pig hearts and ventricular arrhythmia in rabbit hearts. ${ }^{31,32}$ The results of the present study are in line with these earlier investigations. However, it is important to note that the experimental protocol of the present study is more clinically relevant. Ranolazine perfusion after coronary occlusion, not its pretreatment, exerted significant antiarrhythmic effects against ischemic VT/VF. It has been estimated that approximately half of arrhythmic deaths occur within phase 1, and therefore, suppression of late $I_{\mathrm{Na}}$-dependent focal activity by ranolazine should be a promising therapeutic strategy to reduce sudden arrhythmic death during the acute phase of myocardial infarction.

\section{Study Limitations}

First, in optical mapping experiments, BDM was perfused to reduce motion artifact, which is known to affect electrophysiological properties of the ventricular myocardium. ${ }^{33,34}$ In addition, ischemia-dependent metabolic changes and mechanical stretch-associated activities may be considerably modified in hearts without physiological muscle contraction. Second, the automatic nervous system and catecholamines play important roles in the genesis of ischemic arrhythmias, ${ }^{46}$ which cannot be assessed in the present study using isolated Langendorff-perfused hearts. Finally, careful attention must be paid when extrapolating the results obtained from rabbit hearts to human.

\section{Conclusions}

Ranolazine perfusion facilitates termination of ischemic VT/VF in isolated rabbit hearts through the suppression of late $I_{\mathrm{Na}}$-mediated focal activity in the BZ. This result may provide novel insights into the arrhythmogenic mechanism in hearts with regional ischemia, and the effectiveness of ranolazine in the treatment of life-threatening ventricular arrhythmias during the acute phase of myocardial infarction.

\section{Acknowledgments}

This work was supported by research grants from the Ministry of Education, Culture, Sports, Science and Technology of Japan (Grantin Aid for Scientific Research on Innovation Areas 22136010) and the Japan Society for the Promotion of Science (Grant-in-Aid for Scientific Research 15K09078).

\section{Disclosures}

No conflicts of interest declared.

\section{References}

1. Rubart M, Zipes DP. Mechanisms of sudden cardiac death. $J$ Clin Invest 2005; 115: 2305 - 2315.
2. Takii T, Yasuda S, Takahashi J, Ito K, Shiba N, Shirato K, et al; MIYAGI-AMI Study Investigators. Trends in acute myocardial infarction incidence and mortality over 30 years in Japan: Report from the MIYAGI-AMI Registry Study. Circ J 2010; 74: 93 100 .

3. SOS-KANTO Committee. Incidence of ventricular fibrillation in patients with out-of-hospital cardiac arrest in Japan: Survey of survivors after out-of-hospital cardiac arrest in Kanto area (SOS-KANTO). Circ J 2005; 69: 1157-1162.

4. Janse MJ, Wit AL. Electrophysiological mechanisms of ventricular arrhythmias resulting from myocardial ischemia and infarction. Physiol Rev 1989; 69: 1049-1169.

5. Carmeliet E. Cardiac ionic currents and acute ischemia: From channels to arrhythmias. Physiol Rev 1999; 79: 917-1017.

6. Di Diego JM, Antzelevitch C. Ischemic ventricular arrhythmias: Experimental models and their clinical relevance. Heart Rhythm 2011; 8: 1963-1968.

7. Maier LS, Sossalla S. The late Na current as a therapeutic target: Where are we? J Mol Cell Cardiol 2013; 61: 44-50.

8. Antzelevitch C, Nesterenko V, Shryock JC, Rajamani S, Song Y, Belardinelli L. The role of late $I_{\mathrm{Na}}$ in development of cardiac arrhythmias. Handb Exp Pharmacol 2014; 221: 137-168.

9. Belardinelli L, Giles WR, Rajamani S, Karagueuzian HS, Shryock JC. Cardiac late $\mathrm{Na}^{+}$current: Proarrhythmic effects, roles in long QT syndromes, and pathological relationship to CaMKII and oxidative stress. Heart Rhythm 2015; 12: 440-448.

10. Makielski JC. Late sodium current: A mechanism for angina, heart failure, and arrhythmia. Trends Cardiovasc Med 2016; 26: $115-122$.

11. Wagner S, Maier LS, Bers DM. Role of sodium and calcium dysregulation in tachyarrhythmias in sudden cardiac death. Circ Res 2015; 116: $1956-1970$.

12. Antzelevitch C, Belardinelli L, Zygmunt AC, Burashnikov A, Di Diego JM, Fish JM, et al. Electrophysiological effects of ranolazine, a novel antianginal agent with antiarrhythmic properties. Circulation 2004; 110: 904-910.

13. Wu L, Rajamani S, Li H, January CT, Shryock JC, Belardinelli L. Reduction of repolarization reserve unmasks the proarrhythmic role of endogenous late $\mathrm{Na}^{+}$current in the heart. Am J Physiol Heart Circ Physiol 2009; 297: H1048-H1057.

14. Rajamani S, El-Bizri N, Shryock JC, Makielski JC, Belardinelli L. Use-dependent block of cardiac late $\mathrm{Na}^{+}$current by ranolazine. Heart Rhythm 2009; 6: 1625-1631.

15. Antzelevitch C, Burashnikov A, Sicouri S, Belardinelli L. Electrophysiologic basis for the antiarrhythmic actions of ranolazine. Heart Rhythm 2011; 8: 1281-1290.

16. Verrier RL, Kumar K, Nieminen T, Belardinelli L. Mechanisms of ranolazine's dual protection against atrial and ventricular fibrillation. Europace 2013; 15: 317-324.

17. Mason FE, Sossalla S. The significance of the late $\mathrm{Na}^{+}$current for arrhythmia induction and the therapeutic antiarrhythmic potential of ranolazine. J Cardiovasc Pharmacol Ther 2017; 22: $40-50$.

18. Yamazaki M, Honjo H, Nakagawa H, Ishiguro YS, Okuno Y, Amino M, et al. Mechanisms of destabilization and early termination of spiral wave reentry in the ventricle by a class III antiarrhythmic agent, nifekalant. Am J Physiol Heart Circ Physiol 2007; 292: H539-H548.

19. Yamazaki M, Honjo H, Ashihara T, Harada M, Sakuma I, Nakazawa K, et al. Regional cooling facilitates termination of spiral-wave reentry through unpinning of rotors in rabbit hearts. Heart Rhythm 2012; 9: 107-114.

20. Peper K, Trautwein W. The effect of aconitine on the membrane current in cardiac muscle. Pflügers Arch Gesamte Physiol Menschen Tiere 1967; 296: 328-336.

21. Berenfeld O, Pertsov AM. Dynamics of intramural scroll waves in three-dimensional continuous myocardium with rotational anisotropy. J Theor Biol 1999; 199: 383-394.

22. Qu Z, Kil J, Xie F, Garfinkel A, Weiss JN. Scroll wave dynamics in a three-dimensional cardiac tissue model: Roles of restitution, thickness, and fiber rotation. Biophys J 2000; 78: 2761-2775.

23. Nieminen T, Nanbu DY, Datti IP, Vaz GR, Tavares CA, Pegler $\mathrm{JR}$, et al. Antifibrillatory effect of ranolazine during severe coronary stenosis in the intact porcine model. Heart Rhythm 2011; 8: $608-614$.

24. Belardinelli L, Shryock JC, Fraser H. Inhibition of the late sodium current as a potential cardioprotective principle: Effects of the late sodium current inhibitor ranolazine. Heart 2006; 92(Suppl 4): iv6-iv14.

25. Stone PH, Gratsiansky NA, Blokhin A, Huang IZ, Meng L; 
ERICA Investigators. Antianginal efficacy of ranolazine when added to treatment with amlodipine: The ERICA (Efficacy of Ranolazine in Chronic Angina) trial. J Am Coll Cardiol 2006; 48: $566-575$.

26. Wilson SR, Scirica BM, Braunwald E, Murphy SA, Karwatowska-Prokopczuk E, Buros JL, et al. Efficacy of ranolazine in patients with chronic angina observations from the randomized, double-blind, placebo-controlled MERLIN-TIMI (Metabolic Efficiency with Ranolazine for Less Ischemia in NonST-Segment Elevation Acute Coronary Syndromes) 36 Trial. $J$ Am Coll Cardiol 2009; 53: 1510-1516.

27. Kosiborod M, Arnold SV, Spertus JA, McGuire DK, Li Y, Yue $\mathrm{P}$, et al. Evaluation of ranolazine in patients with type 2 diabetes mellitus and chronic stable angina: Results from the TERISA randomized clinical trial (Type 2 Diabetes Evaluation of Ranolazine in Subjects with Chronic Stable Angina). J Am Coll Cardiol 2013; 61: 2038-2045.

28. Scirica BM, Morrow DA, Hod H, Murphy SA, Belardinelli L, Hedgepeth CM, et al. Effect of ranolazine, an antianginal agent with novel electrophysiological properties, on the incidence of arrhythmias in patients with non ST-segment elevation acute coronary syndrome: Results from the Metabolic Efficiency with Ranolazine for Less Ischemia in Non ST-Elevation Acute Coronary Syndrome Thrombolysis in Myocardial Infarction 36 (MERLIN-TIMI 36) randomized controlled trial. Circulation 2007; 116: $1647-1652$

29. Dhalla AK, Wang WQ, Dow J, Shryock JC, Belardinelli L, Bhandari A, et al. Ranolazine, an antianginal agent, markedly reduces ventricular arrhythmias induced by ischemia and ischemia-reperfusion. Am J Physiol Heart Circ Physiol 2009; 297: H1923-H1929.

30. Kloner RA, Dow JS, Bhandari A. The antianginal agent ranola- zine is a potent antiarrhythmic agent that reduces ventricular arrhythmias: Through a mechanism favoring inhibition of late sodium channel. Cardiovasc Ther 2011; 29: e36-e41.

31. Belardinelli L, Liu G, Smith-Maxwell C, Wang WQ, El-Bizri N, Hirakawa R, et al. A novel, potent, and selective inhibitor of cardiac late sodium current suppresses experimental arrhythmias. J Pharmacol Exp Ther 2013; 344: 23-32.

32. Alves Bento AS, Bacic D, Saran Carneiro J, Nearing BD, Fuller $\mathrm{H}$, Justo FA, et al. Selective late $I_{\mathrm{Na}}$ inhibition by GS-458967 exerts parallel suppression of catecholamine-induced hemodynamically significant ventricular tachycardia and T-wave alternans in an intact porcine model. Heart Rhythm 2015; 12: $2508-$ 2514.

33. Lee MH, Lin SF, Ohara T, Omichi C, Okuyama Y, Chudin E, et al. Effects of diacetyl monoxime and cytochalasin $\mathrm{D}$ on ventricular fibrillation in swine right ventricles. Am J Physiol Heart Circ Physiol 2001; 280: H2689-H2696.

34. Lou Q, Li W, Efimov IR. The role of dynamic instability and wavelength in arrhythmia maintenance as revealed by panoramic imaging with blebbistatin vs. 2,3-butanedione monoxime. Am J Physiol Heart Circ Physiol 2012; 302: H262-H269.

\section{Supplementary Files}

\section{Supplementary File 1}

Figure S1. Effects of E-4031 $(0.01 \mu \mathrm{mol} / \mathrm{L})$ on ventricular action potentials (APs) and ischemic ventricular tachycardia/ventricular fibrillation (VT/VF).

Please find supplementary file(s);

http://dx.doi.org/10.1253/circj.CJ-17-0128 